\title{
Una experiencia de clase invertida en el Grado en Filología Hispánica
}

\author{
ANA MANCERA RUEDA \\ Universidad de Sevilla \\ Departamento de Lengua Española, \\ Lingüistica y Teoría de la Literatura \\ anamancera@us.es \\ ORCID: https://orcid.org/0000-0001-5343-4935 \\ D.O.I.: http://dx.doi.org/10.12795/JDU.2018.i01.22 \\ Pp.: $390-410$
}

\section{Resumen}

Durante el segundo cuatrimestre del curso 2017/2018 se ha realizado un ciclo de mejora con los alumnos matriculados en la asignatura Análisis lingüistico de texto del español actual, del Grado en Filología Hispánica. Siguiendo las directrices didácticas de la Red de Formación e Innovación Docente (REFID), la innovación se ha centrado en dos aspectos centrales: el desarrollo de clases invertidas (flipped classroom) impartidas por los propios alumnos y la evaluación mediante portafolio.

Palabras clave Análisis lingüístico de textos del español actual, Grado en Filología Hispánica, Docencia universitaria, Experimentación docente universitaria, Análisis del discurso. 


\section{¿Cómo es la asignatura Análisis lingüísticos de textos del español actual?}

En esta comunicación voy a describir el intento de creación de un entorno natural de aprendizaje crítico (Bain, 2004) mediante el desarrollo de clases invertidas (flipped classroom) en Análisis lingüistico de textos del español actual, una asignatura que he impartido durante el segundo cuatrimestre de 2017/2018 en el cuarto curso del Grado en Filología Hispánica. Sobre esta metodología docente cfr., por ejemplo, Bergmann y Sams (2012), Tourón y Santiago (2015) o Fernández Gámez y Guerra (2016), entre otros.

Uno de los objetivos principales de esta materia, de acuerdo con su proyecto docente, es "proporcionar herramientas prácticas para abordar el análisis lingüístico de textos en lengua española desde una perspectiva pragmática que vincule el análisis de los diferentes niveles lingüísticos con el entorno comunicativo en el que se materializan". Por esta razón se trata de que los alumnos analicen productos discursivos pertenecientes a diferentes tipologías textuales y a todo tipo de variedades de lengua, en relación con el contexto sociocultural en el que se han elaborado. Sin establecer una distinción formal entre teoría y práctica, combinando la enseñanza inductiva con la deductiva.

Análisis lingüistico de textos del español actual es una materia optativa de la que solamente se oferta un grupo, a pesar de que el número de alumnos que se matricularon en ella durante el curso académico 2017/2018 es considerable (al menos, de acuerdo con los estándares de las asignaturas optativas que se imparten en la Facultad de Filología): 61 estudiantes.

A la mayoría ya les di clase en tercero, en la asignatura Análisis del discurso y pragmática del español, y durante el primer cuatrimestre de cuarto, en Gramática española, por lo que conocía los nombres de muchos de ellos. Asimismo, los alumnos estaban familiarizados con mi manera 
de enfocar la docencia, lo que permitió que, ya desde el principio, el ambiente fuera bastante distendido. En Análisis del discurso y pragmática del español puse en práctica durante el curso 2015/2016 un ciclo de mejora, gracias a la inestimable ayuda de mis formadores en el Seminario de profesores universitarios noveles de iniciación, Eduardo García y Ana Rivero. Y, desde entonces, trato de aplicar lo alli aprendido a todas mis clases. En las III Jornadas de Docencia Universitaria, coordinadas por Rafael Porlán y Elisa Navarro, he contado mi experiencia (Mancera, 2016).

Sin embargo, en este caso la disposición del aula no era la más apropiada para prescindir de un modelo docente de carácter transmisivo (De Alba y Porlán, 2017), con multitud de bancas alineadas y dispuestas en dirección a una tribuna elevada en la que se situaba la mesa del profesor y la pizarra. El espacio resultaba muy reducido para tan elevado número de alumnos, por lo que era muy difícil avanzar hacia el centro de la clase o las filas posteriores e interactuar con los estudiantes. Por esta razón, muchas de las actividades tuvimos que realizarlas en los pasillos más próximos al aula e, incluso, para aquellas más lúdicas (por ejemplo, las yincanas) optamos por trasladarnos a los jardines del Rectorado.

\section{¿Qué contenidos quería enseñar?}

Los contenidos conceptuales de la asignatura, tal y como se recogen en el programa docente desde su inclusión en 2009 en el plan de estudios del Grado en Filología Hispánica, se organizan en los siguientes bloques temáticos:

1. El análisis lingüístico de textos: cuestiones metodológicas

2. Las tres dimensiones del texto:

a) Dimensión semántica. El tema: la coherencia y la progresión temática

b) Dimensión sintáctica: elementos lingüísticos y mecanismos de cohesión 
c) Dimensión pragmática y enunciativa. El entorno comunicativo. Aportaciones del Análisis del Discurso y de la Lingüística del Texto

3. Tipología textual: narración, exposición, descripción y argumentación

4. Lengua hablada y lengua escrita. Análisis lingüístico de textos orales y escritos

5. De la teoría a la práctica: comentario lingüístico de textos (gramática, léxico, construcción discursiva y unidades del análisis textual, actos de habla y otras cuestiones)

Sin embargo, al impartir esta asignatura en cursos anteriores me di cuenta de que gran parte del temario se solapaba con el de Análisis del discurso y pragmática del español, una asignatura obligatoria que todos los alumnos habían tenido que cursar ya en el primer cuatrimestre del curso precedente. En cambio, otras cuestiones que hoy se consideran fundamentales en el ámbito del Análisis del discurso quedaban fuera del programa, tanto del de la asignatura Análisis lingüístico de textos del español actual, del que me ocuparé en la presente comunicación, como del de Análisis del discurso y pragmática del español, las dos únicas materias del Grado en Filología Hispánica que se centran en la enseñanza de este tipo de contenidos. Por ejemplo, el estudio de las relaciones discursivas y de las diferentes formas de reproducción del discurso, así como los procedimientos para la expresión de la atenuación lingüística o el análisis de la ironía y el humor, entre otras cuestiones.

Por esta razón, el primer día de clase repartí el siguiente cuestionario para comprobar hasta qué punto los alumnos habían adquirido un aprendizaje significativo de dichas cuestiones pues, de no ser así, volvería a incluirlas entre los contenidos conceptuales que impartiría en las siguientes semanas. Por otra parte, me interesaba conocer qué porcentaje de discentes estaba matriculado en otra 
asignatura optativa que se imparte también en el cuarto curso del Grado en Filología Hispánica durante el segundo cuatrimestre, Español coloquial, con la que pueden llegar a solaparse diversos contenidos. Además, quería saber cuáles eran las expectativas profesionales de los alumnos, al concluir sus estudios. Por último, me interesaba averiguar por qué habían escogido esta asignatura (el número de alumnos matriculados se había incrementado considerablemente en este último curso) y qué esperaban de ella, así como de la propia docente:

1) ¿Cuándo cursaste la asignatura Análisis del discurso y pragmática del español? ¿Quiénes fueron tus profesoras?

2) ¿Has cursado la asignatura Español Coloquial? Si no es así, ¿puedes indicarme si tienes o no la intención de cursarla?

3) ¿Cuándo crees que terminarás tus estudios de grado? (Por favor, haz un cálculo realista, atendiendo a tu trayectoria académica).

4) ¿Qué trabajo te gustaría desempeñar tras acabar tus estudios de grado? ¿Quieres cursar algún máster? ¿Cuál?

5) ¿Te interesaría preparar uno de los temas en grupo y exponerlo en clase para tus compañeros?

6) ¿Qué esperas de esta asignatura? ¿Y de la profesora?

7) ¿Puedes explicar con tus propias palabras qué es la coherencia? ¿Y la cohesión?

8) ¿Qué mecanismos cohesivos recuerdas?

9) ¿Qué tipologías textuales conoces?

10) ¿Es lo mismo argumentar que persuadir? ¿Todos los textos son argumentativos?

11) ¿Puedes resumir las diferencias entre lengua hablada y lengua escrita?

12) ¿Qué es la cortesía?

13) ¿Recuerdas qué es un marcador del discurso? Explícalo con un ejemplo. 
14) ¿Cuál es la diferencia entre locutor, enunciador y sujeto empírico?

15) ¿Has estudiado alguna vez la teoría del humor verbal?

Todos los alumnos que asistieron a clase el primer día completaron el cuestionario, es decir, un total de 48 estudiantes. Para que pudieran responder con total libertad, les animé a utilizar un seudónimo en lugar de su nombre y les pedí que estuvieran lo suficientemente familiarizados con él como para recordarlo al final de curso, a la hora de completar el cuestionario final de la asignatura.

La lectura de las respuestas de los alumnos al cuestionario me convenció de que era necesario reformular algunos de los aspectos del mapa de contenidos que había impartido en los cursos precedentes. De hecho, una de las cuestiones que aprendi en el Seminario de profesores universitarios noveles de iniciación es que es necesario tener en cuenta la jerarquía de los contenidos conceptuales incluidos en el temario de una asignatura. Esto es algo que han puesto también de manifiesto García Díaz, Porlán y Navarro (2017). Todo ello me ha llevado a replantearme el proyecto docente, poniendo el foco en aquellos contenidos esenciales para el perfil profesional del Grado en Filología Hispánica, y a dejar de lado otros aspectos que podríamos calificar de secundarios para la formación del alumno. Y es que coincido con Martín del Pozo, Pineda y Duarte (2017: 30) en que uno de los principios fundamentales para una formación coherente con un modelo de enseñanza centrado en el estudiante es la "organización de la formación en torno a problemas profesionales relevantes vinculados a la práctica docente". En la figura 1 se presenta el mapa conceptual de los contenidos de la asignatura, que organicé finalmente en catorce temas articulados en torno a las cinco nociones esenciales recogidas en el programa: 


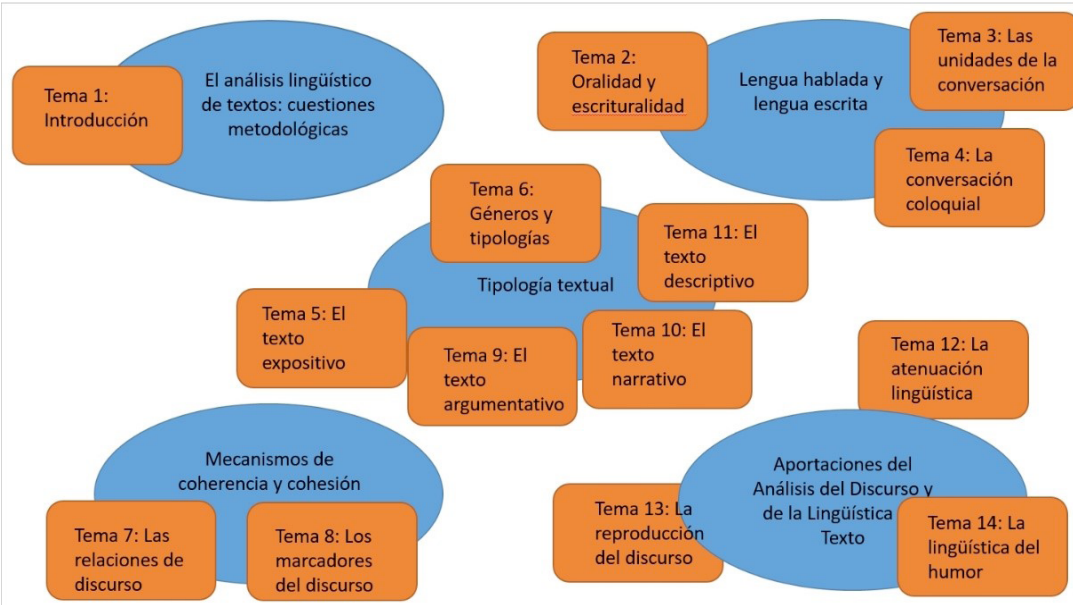

Figura 1: Mapa de contenidos de la asignatura Análisis lingüístico de textos del español actual impartidos durante el curso 2017/2018.

Fuente: elaboración propia.

Hay que tener en cuenta, además, que cualquier modificación sustancial de los contenidos impartidos en una asignatura del Grado en Filología Hispánica de la Universidad de Sevilla deben ser sometidos a una evaluación por parte de la Agencia Nacional de Evaluación de la Calidad y Acreditación (ANECA).

Por otra parte, en los últimos años me he percatado de que los alumnos que están concluyendo el Grado en Filología Hispánica, en general, poseen gran temor a hablar en público, su competencia de expresión oral no es excesivamente elevada y se encuentran con graves problemas a la hora de tratar de comprender por sí mismos, sintetizar y explicar aspectos clave de la bibliografía especializada sobre Análisis del discurso. Pienso que esto puede suponer un grave lastre para su incorporación al mercado laboral. Por ejemplo, como docentes en la enseñanza secundaria o en la universidad, o como profesores de español para extranjeros (estas son las salidas profesionales a las que aspiraba la mayoría, a juzgar por sus respuestas a la pregunta 4 del cuestionario inicial). 
Por esta razón, con el diseño de este "ciclo de mejora de asignatura completa" (Porlán, 2016) pretendí que fueran los propios alumnos los que, en grupos reducidos, llevaran a cabo la exposición de los contenidos del temario. Esta organización de la docencia en clases invertidas permitiría que los estudiantes adquirieran también contenidos actitudinales y procedimentales relacionados, por ejemplo, con la capacidad de trabajar en equipo, de elaborar una unidad didáctica, de exponer en clase los resultados de su propio análisis de textos de distinto tipo, de conocer los modelos mentales de sus compañeros y relacionarlos con los suyos, de explicar de manera sencilla nociones complejas vinculadas con los saberes principales de la disciplina, de mejorar sus competencias de expresión oral y de comprensión auditiva, etc. En cuanto a mi papel, desde el principio tuve claro que debía abandonar la posición elevada que me confería la tarima (tanto en sentido literal como metafórico), y actuar como orientadora del proceso de construcción de conocimiento de mis estudiantes (De Alba y Porlán, 2017).

\section{¿Qué ocurrió durante las clases?}

Con el fin de mostrar a los alumnos cómo quería que ellos mismos enfocaran las exposiciones de sus temas en las clases invertidas y proporcionarles tiempo suficiente para planificarlas, los tres temas iniciales fueron expuestos por mí durante las primeras tres semanas. Siguiendo a Bain (2004), intenté identificar los modelos mentales de los estudiantes a la hora de analizar textos que tenían que ver con la actualidad política y social del momento, y de ponerlos frente a informaciones, evidencias y argumentos que supusieran un desafío intelectual para ellos. Por ejemplo, mediante el análisis de las implicaturas existentes en la siguiente viñeta de Forges: 


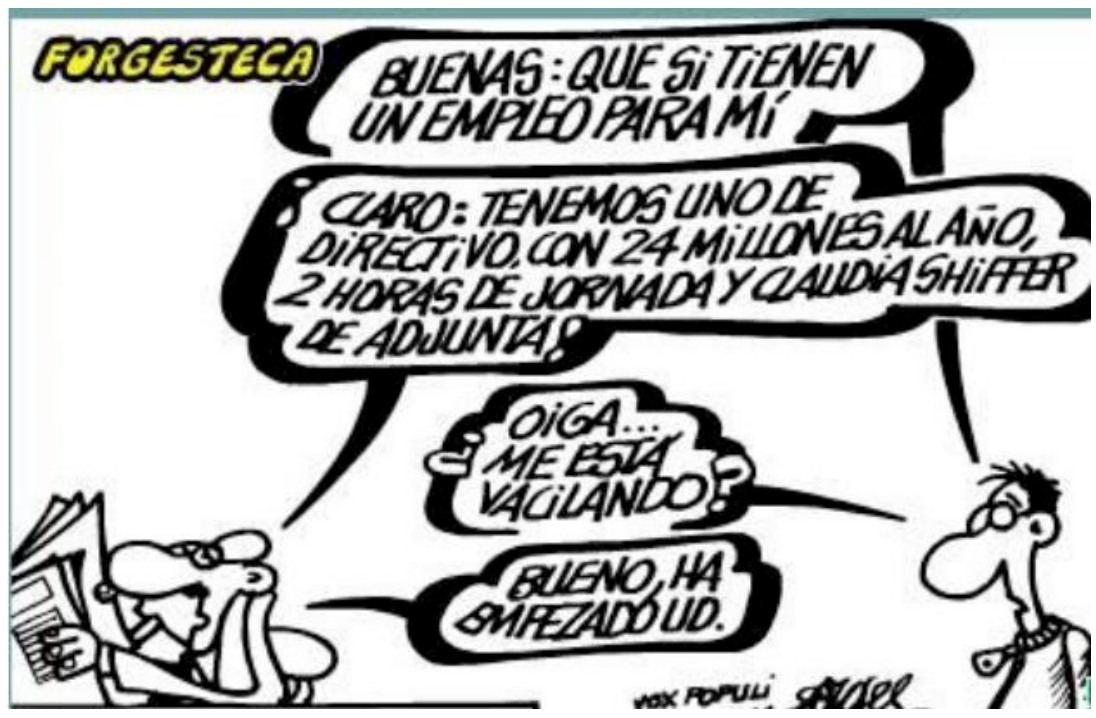

Figura 2: Viñeta del humorista Forges publicada en el diario El País.

Ya desde el primer día de clase intenté que los estudiantes abandonaran su papel habitual de "consumidor pasivo" (Rivero y Porlán, 2017), como meros oyentes de los contenidos transmitidos por el profesor, y que adoptaran un rol más participativo, analizando en grupo y de la manera más pormenorizada que les fuera posible esta viñeta. Se trataba de lograr un "aprendizaje construido" (Pozo, 2008) acerca de aspectos fundamentales para el Análisis del discurso que este texto de Forges permitía abordar de forma amena (como la ironía y el humor, la polifonía o el lenguaje juvenil), pero de manera inductiva (desde la práctica hacia la teoría), y tratando de dar sentido a los contenidos que poco a poco fui suministrándoles a partir de sus propios modelos mentales.

Precisamente escogí esta viñeta porque me permitía relacionar algunos de los contenidos que habíamos visto en tercero, en la asignatura Análisis del discurso y pragmática del español, con la introducción de algunos de los contenidos conceptuales que abordaríamos en las siguientes semanas. Además, muchos se sintieron identificados con el protagonista de la viñeta, el joven que busca empleo, lo 
que dio pie a que comentáramos en clase qué tipo de mecanismos lingüísticos podrían utilizar ellos mismos en una situación comunicativa similar, para lograr su propósito de conseguir un trabajo (algo que sé que les provoca una gran inquietud).

Otra de las actividades que les propuse fue la de organizar la clase en grupos, con el objetivo de elaborar distintos tipos de textos: una carta de motivación para poder estudiar en una universidad extranjera, un correo electrónico a un amigo de la infancia con el que no tenían relación desde hacía mucho tiempo, una petición de matrimonio, un diálogo que mostrara cómo se dirigirian al rey Felipe VI si se encontraran con él en un restaurante, etc. Con esto conseguí, por una parte, fomentar la motivación del alumnado (Finkel, 2000) mediante el desarrollo de su creatividad, haciéndoles redactar textos de muy distinto tipo que luego tuvieron que leer y que representar (en el caso de los diálogos) ante sus compañeros. Por otra parte, esto nos dio pie a trabajar con distintos aspectos vinculados con las selecciones léxicas más acordes con diversos tipos de registros, las estrategias discursivas, la distinción entre el discurso hablado y el escrito desde el punto de vista concepcional y medial, los aspectos universales e idiomáticos de la lengua hablada, las condiciones comunicativas y las estrategias de verbalización del habla frente a la escritura, etc.

Durante la tercera semana de clase nos centramos en la conversación coloquial. Para explicar la alternancia de turnos y la distribución de las interacciones conversacionales en pares adyacentes me serví de este conocido diálogo de los humoristas Tip y Coll, que comentamos tras contemplarlo en vídeo: 
COLL: -Muy buenas, ¿tiene usted cerillas?

TIP: -Muy buenas.

COLL: -Muy buenas, ¿tiene usted cerillas?

TIP: -Muy buenas.

COLL: -Muy buenas, ¿tiene usted cerillas?

TIP: -Muy buenas.

Figura 3: Transcripción de un fragmento de uno de los diálogos de los humoristas Tip y Coll

Para la exposición de los temas 4-14 organicé once grupos de trabajo con un máximo de cuatro integrantes. Cada alumno pudo elegir libremente en qué grupo integrarse con el fin de exponer uno de los temas, conforme a la planificación docente prevista, dedicando un máximo de tres clases a la explicación de cada tema.

La bibliografía para la preparación de los temas se la suministré yo misma, escaneando un buen número de artículos que puse a disposición de todos los estudiantes en mi página web. Al comenzar a plantear este ciclo de mejora dudé acerca de si debía conceder a los discentes total autonomía para la búsqueda bibliográfica y la estructuración de cada uno de los temas, pero finalmente decidí no hacerlo así, puesto que la bibliografía disponible sobre algunas cuestiones es excesivamente amplia y pensé que mis alumnos, que solo habían cursado con anterioridad una asignatura sobre Análisis del discurso, no estaban lo suficientemente preparados como para discernir las aportaciones más significativas.

Jornadas de Formación e Innovación Docente del Profesorado I № 1 (2018) Esta obra se distribuye con la licencia Creative Commons 


\section{¿Qué aprendieron mis estudiantes durante las clases invertidas?}

Entre las competencias que adquirieron la mayor parte de mis alumnos a lo largo de las clases invertidas cabe destacar las siguientes:

1. La capacidad de comprender por sí mismos la bibliografía especializada. En diversas tutorías fui ayudándoles a resolver sus dudas, pero tras una fase previa en la que les insté a que fueran ellos mismos los que "se enfrentaran" directamente a la labor de "descifrar" la bibliografía (como a muchos de ellos les gustaba decir). Según me han comentado algunos en el portafolio, "este entrenamiento" les ha resultado especialmente útil para abordar su trabajo de fin de máster.

2. El aprendizaje de las destrezas necesarias para poder trabajar en equipo, ya que la exposición de un tema era algo que todos los miembros de un grupo debían preparar conjuntamente. Asimismo, todos los integrantes de un equipo tenían que estar presentes y colaborar con los demás durante la explicación del tema a lo largo de las tres clases. A pesar de que a veces surgieron pequeños conflictos, muchos alumnos reconocieron al concluir el periodo docente la importancia de las dinámicas de trabajo que habían conseguido desarrollar, por ejemplo, al poner en común lo que cada uno interpretaba al leer la bibliografía, o al darse cuenta de las distintas visiones que tenían acerca de una misma realidad (Finkel, 2000).

3. La capacidad de sintesis, ya que la exposición de cada tema no podía alargarse más de tres clases. Esto les obligaba a resumir la bibliografía que yo les había proporcionado, seleccionando los contenidos fundamentales, organizándolos 
en distintos epígrafes y reelaborando cada una de sus sesiones en función del tiempo disponible. Algunas presentaciones fueron incluso excesivamente sintéticas, pero el tiempo sobrante lo dediqué a la tutorización grupal y al trabajo individual en torno a las cuestiones del portafolio. 4. Dado que la asignatura se denomina Análisis lingüistico de textos del español actual, para mí era muy importante que los estudiantes no se limitaran a presentar los contenidos de la bibliografía, sino que aplicaran también la teoría al análisis de diversos tipos de textos. Por eso debían buscar ejemplos propios para ilustrar los distintos aspectos teóricos (al menos, el 80\% de los ejemplos tenían que haber sido localizados por los propios alumnos) y proponer algunas actividades para realizar durante las clases. No se trataba simplemente de presentar todos los conceptos, tal y como aparecían en la bibliografía, sino de hacerlos comprensibles para el resto de los compañeros. Era importante que los contenidos se trabajaran en este sentido, buscando ejemplos adecuados para ilustrar los distintos aspectos teóricos. Sin duda, este ha sido uno de los grandes aciertos de la asignatura, pues el tener que buscar ejemplos propios les Llevaba a realizar una lectura más atenta de la bibliografía, a evaluar la adecuación de textos de distinto tipo para su exposición, a tratar de localizar muestras de lengua que fueran interesantes y que resultaran amenas para sus compañeros. Por ejemplo, fragmentos de sus obras literarias favoritas, monólogos extraídos del programa El club de la comedia, de películas de los Hermanos Marx, de series de televisión, vídeos de sus youtubers preferidos, chistes, tiras cómicas, mensajes de WhatsApp, tuits, memes, etc. 
Además, a medida que avanzó el cuatrimestre y que se fue afianzando el grado de confianza entre los integrantes de la clase, muchos grupos se atrevieron a combinar lo didáctico con lo lúdico desarrollando secuencias didácticas en las que insertaban cada vez más juegos con los que afianzar la comprensión de los aspectos teóricos: concursos basados en la herramienta digital Kahoot, debates sobre cuestiones hilarantes para poner en práctica los mecanismos argumentativos aprendidos, representaciones teatrales con las que cada grupo exhibía su creatividad literaria, actividades en las que debían completar los marcadores discursivos presentes en la letra de diversas canciones, yincanas, etc.

5. El desarrollo de la competencia digital y la capacidad de ajustarse a los plazos establecidos. Cada grupo tenía que crear una presentación en power point con los textos y documentos en audio y vídeo que iban a presentar y remitírmela, al menos, una semana antes de su exposición en clase, con el fin de que yo misma pudiera supervisarla. Asimismo, la versión definitiva de esta presentación debían enviármela el lunes de la semana siguiente a su exposición, para que pueda subirse a la página web de la asignatura y servir de ayuda en la realización de las actividades del portafolio. Aunque no todos los grupos han sido igual de cumplidores con los plazos, en general, estoy satisfecha con este aspecto.

6. El desarrollo de la competencia de expresión oral y la capacidad de hacer comprensibles para los compañeros de clase contenidos de cierta complejidad. Hemos trabajado mucho esta cuestión en las tutorías con algunos grupos, pero he de reconocer que, a pesar de mis indicaciones, a la hora de realizar su presentación algunos 
alumnos se limitaron a leer el power point y a reproducir las dinámicas docentes de la clase magistral prototípica. Esto ha sido muy criticado por sus compañeros en la evaluación por pares que han tenido que realizar en el portafolio.

7. El desarrollo de la competencia de comprensión auditiva y el estímulo del espíritu crítico: en primer lugar, al tener que confrontar planteamientos muy distintos de autores diversos; en segundo lugar, tras cada una de las sesiones, al valorar la exposición de los compañeros ofreciendo propuestas de mejora con los hashtags \#asísí, \#asíno, \#ysi..., un recurso inteligente para ofrecer retroalimentación que Juan Pablo Mora me enseñó a incorporar en todas mis clases y que también me ha permitido aprender mucho a través de los juicios críticos de mis alumnos; por último, en la evaluación final, pues las exposiciones de cada grupo fueron también calificadas por los propios alumnos.

\section{¿Cómo fue la evaluación?}

Como explican Rivero y Porlán (2017: 74), "el análisis de la información recogida durante la evaluación nos permite emitir juicios fundamentados sobre el grado en que el aprendizaje y la enseñanza alcanzan las finalidades que nos hemos planteado". De los instrumentos de evaluación que estos autores identifican yo me he servido fundamentalmente de tres: el portafolio o carpeta de aprendizaje, la observación de todo lo que sucedía en el aula y durante las tutorías, y las TIC.

Durante la primera semana de clase ofrecí a todos los alumnos la posibilidad de escoger entre dos sistemas de evaluación:

a) Examen escrito: $70 \%$ de la calificación final. 
- Trabajo individual de análisis de un texto: 20\% de la calificación final.

- Participación y asistencia a clase: $10 \%$ de la calificación final.

b) Exposición grupal en clase y portafolio: $80 \%$ de la calificación final

- Trabajo individual de análisis de un texto: 20\% de la calificación final.

- En este segundo sistema de evaluación la asistencia al $80 \%$ de las clases será obligatoria.

La mayor parte de los alumnos escogió el sistema b), pues le permitía prescindir del examen. Únicamente dos estudiantes Erasmus decidieron presentarse al examen, pues la exposición grupal les provocaba cierta ansiedad. Dada la naturaleza de esta asignatura, en la que lo fundamental es que el alumno se familiarice con el análisis de diversos tipos de textos desde perspectivas muy diferentes, no me parecía esencial que estos se limitaran a estudiar una información sobre tal o cual perspectiva que luego tendrían que "volcar acríticamente" en las hojas del examen. Por el contrario, pensé que sería más apropiado para su formación que ellos pusieran en práctica el análisis desde todas las perspectivas explicadas y de, al menos, un texto correspondiente a todas las tipologías mencionadas en clase. Contando con tiempo suficiente para la reflexión crítica. Por esta razón permití a los alumnos entregarme el portafolio en una fecha posterior a la finalización del período de exámenes de junio. Sin embargo, a lo largo del cuatrimestre, les fui aconsejando que realizaran la actividad del portafolio correspondiente a cada tema tras la explicación del mismo en clase.

El portafolio debía realizarse de manera individual, ya que para mí era importante que cada alumno adquiriera las competencias de expresión y la capacidad analítica que las actividades propuestas en él conllevaban.

Jornadas de Formación e Innovación Docente del Profesorado I № 1 (2018) Esta obra se distribuye con la licencia Creative Commons 
En primer lugar, el portafolio constaba de un "semanario del alumno" en el que este debía realizar un resumen valorativo de los aspectos fundamentales de cada tema, indicando también qué sesiones les habían interesado más. En segundo lugar, tenía que incluirse una valoración mediante rúbricas de las exposiciones de los distintos grupos, con una calificación numérica acompañada de la justificación de los aspectos positivos y de aquellas cuestiones mejorables. Esta debía acompañarse por una autoevaluación acerca del trabajo personal realizado.

La parte más extensa del portafolio era la constituida por las actividades propuestas por mí para aplicar lo aprendido en cada uno de los temas. Estas se recogen a continuación:

a) Transcribe una conversación utilizando el sistema del grupo Val.Es.Co. (máximo: 3 minutos) y analiza en ella las constantes y estrategias del registro coloquial (temas 1-4).

b) Escribe un texto expositivo acerca de un concepto fundamental en el ámbito de la Pragmática o del Análisis del discurso (recuerda que está prohibido el plagio, es decir, debes consultar la bibliografía pertinente), como si fueras a publicarlo en Wikipedia (máximo: 700 palabras). Analiza tu texto de acuerdo con las características del discurso expositivo (tema 5).

c) Escribe una reseña crítica acerca del contenido de los dos textos que conforman el tema 6 (máximo: 1.000 palabras). Analiza este género discursivo en función de la situación de comunicación.

d) Escribe una breve columna de opinión sobre el tema que desees (mínimo: 500 palabras) y analiza en ella las relaciones del discurso (tema 7).

e) Imagina que estás escribiendo una novela. Inventa un diálogo entre varios de tus personajes (máximo: 1.000 palabras). Analiza los marcadores discursivos que utilizan (tema 8). 
f) Redacta una carta de motivación ensalzando los principales aspectos de tu currículo y tus maravillosas cualidades para poder acceder a un máster o para conseguir el trabajo o las prácticas "de tus sueños" (mínimo: 500 palabras). Analiza en ella los recursos argumentativos que has utilizado (tema 9).

g) Escribe un relato (máximo: 1.000 palabras). Analiza el narrador y los modos del discurso narrativo que hayas utilizado (tema 10).

h) Redacta una descripción física y psicológica de ti mismo (máximo: 1.000 palabras). Analízala de acuerdo con las características del texto descriptivo (tema 11).

i) Imagina que has ido a comer a un restaurante o que has pasado la noche en un restaurante. Escribe una reseña crítica para TripAdvisor contando tu experiencia. Debes servirte de diversos procedimientos de atenuación lingüística para mostrar tu opinión sin resultar excesivamente descortés (máximo: 1.000 palabras). Posteriormente, realiza un análisis de dichos procedimientos atenuativos (tema 12).

j) Imagina que eres periodista y que tienes que redactar una crónica para tu diario contando una buena noticia de trascendencia internacional (máximo: 1.000 palabras). Utiliza distintos procedimientos de cita para introducir enunciados de discurso referido y analízalos. Explica también las características del discurso reproducido (tema 13).

k) Explica la Teoría General del Humor Verbal sirviéndote de una selección de tuits (tema 14).

Con este tipo de actividades, además de instarles a poner en práctica el análisis de textos de muy distinto tipo de acuerdo con las perspectivas explicadas en cada uno de 
los temas, trataba de estimular también la creatividad de los alumnos. Algo que ha entusiasmado a muchos de ellos, que poseen inquietudes literarias.

Por otra parte, independientemente de que los alumnos hubieran seleccionado el sistema de evaluación a) o b), todos ellos debían realizar el análisis detallado de un texto escogido por ellos mismos siguiendo alguna de las perspectivas presentadas en el temario. Esta actividad tenían que realizarla también de manera individual, pues es la única forma de demostrar que cada uno sabe aplicar correctamente lo explicado en clase al análisis de un texto completo, y de carácter más extensa. Aunque yo misma les estuve asesorando durante las tutorías, considero que la selección personal del texto que cada uno desee analizar, así como de la perspectiva más propicia para abordar tal tipo de análisis, ya conlleva un aprendizaje.

Las TIC me han ayudado también a evaluar el aprendizaje de los alumnos. Por una parte, mediante la corrección de las presentaciones en power point que cada grupo iba enviándome con anterioridad a su exposición. Asimismo, la posibilidad de realizar cuestionarios con la herramienta Kahoot, me permitía comprobar qué aspectos no habían quedado claros de las exposiciones e insistir en ellos al final de la última de las sesiones de cada uno de los temas.

Por último, la observación atenta de lo que sucedía tanto en las clases como durante las tutorías grupales me permitía tomar notas mediante palabras clave sobre aspectos que no quedaban claros, con el fin de retomarlos en una sesión posterior o de abordarlos con el resto de la clase (en el caso de las tutorías).

\section{¿Cuáles son mis principios didácticos después de este ciclo de mejora?}

Aunque al principio abordé este ciclo de mejora con cierta inquietud, pues suponía un salto cualitativo en mi trayectoria como docente, tengo que reconocer que esta 
experiencia me ha resultado muy positiva. Con frecuencia, los profesores estamos más preocupados por transmitir correctamente una determinada información que por entender por qué los estudiantes no la comprenden (Sanmartí, 2012). Pero, en esta ocasión, traté de abandonar por completo el modelo didáctico meramente transmisivo, para otorgar protagonismo a los propios alumnos, aunque sin olvidar los contenidos de la enseñanza (García Pérez y Porlán, 2017). Poniendo el foco, no tanto en el "saber", como en el "saber hacer". En este caso, el saber analizar críticamente cualquier tipo de texto. Mediante las clases invertidas, intenté favorecer un "aprendizaje autodirigido" (Marcelo, 2010), potenciando la autonomía y la responsabilidad de los estudiantes, así como el trabajo colaborativo y el sentido crítico.

\section{Referencias bibliográficas}

Bain, K. (2004). What the Best College Teachers Do. Cambridge: MA. Harvard University Press. (Trad. Cast.: Lo que hacen los mejores profesores universitarios. Valencia: Publicaciones de la Universidad de Valencia, 2007. 2a edición).

Bergmann, j. y Sams, A. (2012). Flip your Classroom: REach Every Student in Every Class Every day. Washington, DC: ISTE y Alexandria, VA: ASCD.

De Alba, N. y Porlán, R. (2017). La metodología de enseñanza. En Porlán Ariza (Coord.) Enseñanza universitaria. Cómo mejorarla (pp. 37-53). Madrid: Morata.

Fernández Gámez, D. y Guerra, M. D. (2016). Aprendizaje inverso en formación profesional: opiniones de los estudiantes. International Journal of Technology and Educational Innovation, 2(1), 29-37.

Finkel, D. (2000). Teaching with Your Mouth Shut. Porstmouth: NH. Heinemann Boynton/Cook. (Trad. cast.: Dar clase con la boca cerrada. Valencia: Publicaciones de la Universidad de Valencia, 2008).

García Díaz, E., Porlán, R. y Navarro, E. (2017). Los fines y los contenidos de enseñanza. En Porlán Ariza (Coord.) Enseñanza universitaria. Cómo mejorarla (pp. 55-72). Madrid: Morata. 
García Pérez, F. F. y Porlán, R. (2017). Los Principios Didácticos y el Modelo Didáctico Personal. En Porlán Ariza (Coord.) Enseñanza universitaria. Cómo mejorarla (pp. 93-104). Madrid: Morata.

Mancera, A. (2016). Enseñar Pragmática mediante la elaboración y el análisis de textos: una experiencia docente en el Grado en Filología Hispánica. En Porlán Ariza y Navarro Medina (Coords.) III Jornadas de Docencia Universitaria. Universidad de Sevilla (30 de junio y 1 de julio de 2016) (pp. 973-985). Sevilla: Universidad de Sevilla.

Marcelo, C. (2010). Autoformación para el siglo XXI. En Gairín Sallan (Coord.) Nuevas estrategias formativas por las organizaciones (pp. 141-170). Madrid: Wolters Kluwer.

Martín del Pozo, R., Pineda, J. A. y Duarte, O. (2017). La formación docente del profesorado universitario. En Porlán Ariza (Coord.) Enseñanza universitaria. Cómo mejorarla (pp. 24-36). Madrid: Morata.

Porlan, R. (2016). Programa integrado de Formación e Innovación docente centrada en el aula (FIDA): un objetivo estratégico para el III Plan Propio de Docencia. Sevilla: Universidad de Sevilla.

Pozo, J. I. (2008). Aprendices y maestros. Madrid: Alianza Editorial.

Rivero, A. y Porlán, R. (2017). La evaluación en la enseñanza universitaria. En Porlán Ariza (Coord.) Enseñanza universitaria. Cómo mejorarla (pp. 73-91). Madrid: Morata.

Sanmartí, N. (2012). Evaluar para aprender. 10 ideas claves. Barcelona: Graó.

Tourón, J. y Santiago, R. (2015). El modelo Flipped Learning y el desarrollo del talento en la escuela. Revista de Educación, 368, 196-231.

Jornadas de Formación e Innovación Docente del Profesorado | № 1 (2018) Esta obra se distribuye con la licencia Creative Commons 